\title{
FRONTO-ETHMOIDAL MUCOCOELE AS A CAUSE OF UNILATERAL PROPTOSIS*†
}

\author{
BY \\ P. W. R. M. ALBERTI, H. F. MARSHALL, AND J. I. MUNRO BLACK \\ Royal Victoria Infirmary, Newcastle upon Tyne
}

MUCOCOELE of the frontal or ethmoidal sinuses is an uncommon cause of unilateral proptosis which may produce considerable difficulty in diagnosis. There seems a natural tendency for these cases to be sent to an eye specialist, and a curious reluctance for him to refer the patient to an E.N.T. surgeon, often preferring a neurological opinion which may involve the patient in expensive and unpleasant investigations before the true nature of the condition becomes apparent on clinical grounds. We therefore thought it worth while to describe ten recent patients with this condition in an endeavour to establish more firmly the diagnostic criteria.

Mucocoeles of the paranasal sinuses were first described by Langenbeck (1820) under the name of hydatides, and Rollet (1909) suggested the name mucocoele. The condition aroused interest in the earlier part of the 20th century; Logan Turner (1907) gave a comprehensive account of the condition, and Gerber (1909) assembled and published 178 cases. Howarth $(1921,1924)$ made it the subject of a Hunterian lecture and Lambert (1955) described mucocoele of the frontal or ethmoidal air sinuses as the most common nasal condition to produce proptosis; he described nine patients seen in his department between 1949 and 1953 with chronic nasal mucocoele.

\section{Definition}

Mucocoeles are collections of mucus enclosed in a sac of lining sinus epithelium within an air sinus resulting from an obstruction to the outlet of the cavity which may cause an expansion of the sinus by resorption of the bony walls. The condition commonly occurs in the frontal or ethmoidal group of sinuses and is rarely found as an isolated intranasal lesion within the confines of the middle turbinate. The sac may be filled with pus as a result of chronic infection, in which event it is known as a chronic pyocoele; this differs from an acute infection in a mucocoele which may produce the dramatic signs of a ruptured sinus which are outside the scope of this communication.

\section{Aetiology}

Gerber (1909) suggested that pre-existing catarrh was one cause of the chronic inflammatory changes in the sinuses which led to a temporary or permanent obstruction of the ostium, which was then followed by a gradual accumulation of mucus within the cavity and

* Received for publication January 24, 1968.

+ Address for reprints: E.N.T. Department, Royal Victoria Infirmary, Newcastle-upon-Tyne 1. 
secondary expansion and absorption of the bony walls. Howarth (1921) emphasized that pre-existing trauma to the orbit or nose might be a significant factor in the pathogenesis of the condition, although there might be a considerable time interval, varying from months to years, between the traumatic incident and the onset of proptosis. He also suggested that osteoma within the frontal sinus might obstruct the duct and lead to the production of a mucocoele; he described one such case and cited others.

In three of our patients, there was a history of preceding long-standing sinus disease, and in our view recurrent infection and inflammation produced a block of the openings of the involved cells. When the lesions in these patients were explored, they were found to be chronic pyocoeles, and it may be argued that they were pyocoeles from the start, rather than mucocoeles which became infected. In one young patient with fibrocystic disease, the abnormally viscid nature of the mucus produced in the sinuses could have led to inspissation and obstruction of a duct. Neither trauma nor osteoma was a significant feature in our patients. Inflammation and infection in a pre-existing small mucocoele may be followed by quite rapid enlargement of the swelling and lead the patient to seek attention after a very long preceding history. In such patients, although pus may be obtained from the cyst, we feel that the infection is secondary in a mucocoele. Likewise, one patient was incorrectly diagnosed as having a cyst of the lacrimal sac, and this was explored by an ophthalmic surgeon who aspirated clear mucus. By the time definitive surgery was carried out a pyocoele was removed, but this was a secondary infection following the previous surgery.

\section{Clinical Features}

The patients in this series comprise eight adult women, one adult male, and a child aged $2 \frac{1}{2}$ years. The ages of the adults ranged from 23 to 74 years but commonly the condition was first noticed in young adult life. The preponderance of women is unusual as the other reported series indicate an equal sex ratio. The common presenting complaint was a longstanding orbital swelling producing displacement of the globe; six patients gave a history of proptosis which had been present for more than 6 months at the time of the initial consultation and, in two instances, the history was longer than 10 years.

\section{Case Reports}

Case 1, a middle-aged woman, had first become aware of a right-sided proptosis 20 years previously, and this had slowly progressed until it suddenly increased in size during an upper respiratory infection. It was so gross that she could no longer wear spectacles, and she was referred to an ophthalmic surgeon who immediately referred her to one of us (M.B.). She had a marked downwards, forwards, and outwards proptosis and a soft, fluctuant swelling above and medial to the right eye (Fig. 1, opposite).

Radiological examination confirmed the clinical impression of a fronto-ethmoidal mucocoele. Intranasal examination revealed a swollen bulla ethmoidalis, and needle exploration produced thick, glutinous mucus. This was cured by intranasal opening under local anaesthesia.

Case 2, a woman aged 54, had had an obliterative osteoplastic operation in her left frontal sinus 10 years before, after which she had been well for some years, but then again developed the symptoms of chronic sinusitis. This was followed by a marked left-sided proptosis, present for some months, which led to her referral. There was a palpable swelling in the medial aspect of the left orbital margin, which was soft and fluctuant. On exploration a chronic pyocoele was found to extend along the whole length of the orbital roof, medially into the ethmoid labyrinth, and into the nose. Interestingly, the frontal sinus contained air. 
FIG. 1.-Downwards, forwards, and outwards proptosis caused by a fronto-ethmoidal mucocoele.

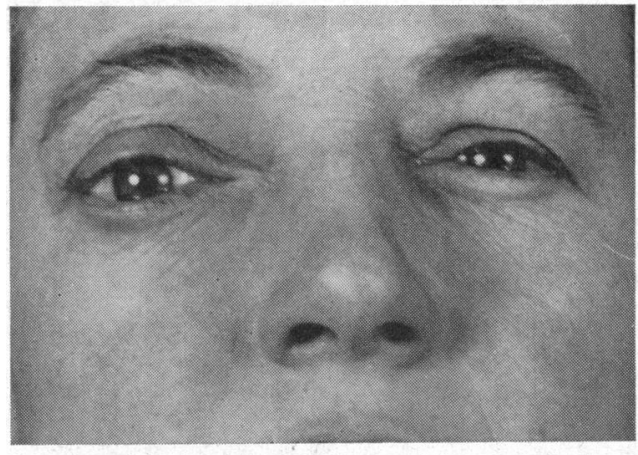

A preceding history of paranasal sinus disease was given by three patients, one of whom had had major sinus surgery performed several years previously.

Even if there is no preceding history of sinusitis, the patients often describe an increase in the degree of proptosis during the course of a cold, which then may reduce again as the infection subsides.

A mucocoele is an extremely unusual cause for proptosis in an infant. It is therefore not surprising that when one presented it caused considerable diagnostic difficulty.

Case 3, a baby girl aged $2 \frac{1}{2}$ years who had cystic-fibrosis, was referred by her paediatrician to an ophthalmic surgeon at the age of 18 months with a one year history of left epiphora. A diagnosis of blocked nasolacrimal duct was made and the duct was dilated with no improvement. Shortly thereafter the child developed a proptosis, which slowly progressed over the next 10 months when $x$-ray examination of the sinuses suggested a cyst of the ethmoid labyrinth. She was referred to Mr. J. D. K. Dawes, who explored the mass through a Howarth incision and found a chronic pyocoele extending from the antrum to the orbital apex and exposing the dura of the anterior fossa. The direction of the proptosis in the child was lateral, slightly forwards and upwards. In spite of the pus in the cyst, there was no history of preceding acute infection.

Three patients had marked epiphora and were diagnosed as and treated for cysts of the lacrimal sac for several months before the true nature of the condition was recognized and they were referred to an otolaryngologist.

A soft fluctuant palpable swelling was present in six of nine patients whose records are available; it was usually situated at the upper medial margin of the orbit, although on one occasion when the cyst arose in a lateral extension of the frontal sinus the swelling occurred at the upper lateral margin of the orbit. The skin always moved readily over the swelling but there was occasionally an associated slight puffiness of the upper eyelid. Palpable deficiencies in the orbital margin above the swelling are a frequent occurrence, but we have not found the hyperostosis at the edges of the deficiency which has been described as characteristic of the lesion (Vail, 1931).

A noteworthy feature of this group of patients is the relative lack of any symptoms produced by the lesion, in spite of the very large size that some mucocoeles may attain (Fig. 2, overleaf).

Oculomotor palsies are not a feature of orbital mucocoeles and diplopia is rare in spite of the considerable displacement of the globe which may occur. It was in fact found in only one of our patients, a girl with a massive displacement of the globe, and even then it was only present on lateral gaze and cleared quickly after treatment of the mucocoele. Visual field 


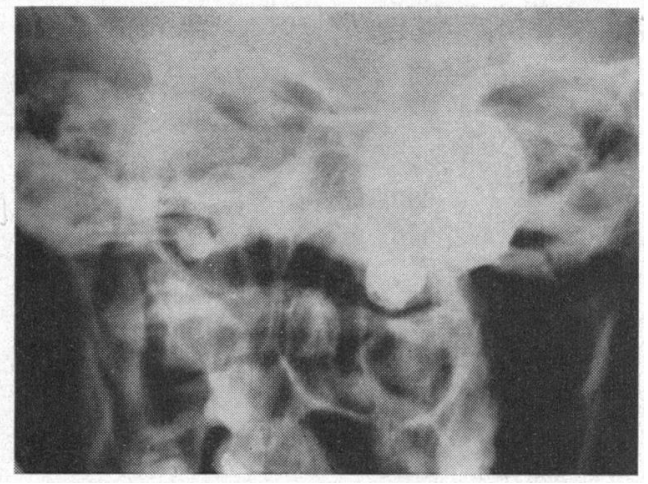

Fig. 2.-Antero-posterior $x$ ray of skull, showing extensive mucocoele cavity filled with radio-opaque gauze.

defects are likewise extremely uncommon, being found only in large mucocoeles of the posterior ethmoid cells; if present they may lead to considerable diagnostic difficulties.

Case 4, a 56-year-old man, was referred by a neurologist with a 4-month history of left-sided proptosis and a central scotoma. The direction of the proptosis was purely forwards and there was no palpable swelling in the orbital margin. He had already been extensively investigated and the only positive finding was a radiological diagnosis of a tumour or mucocoele in the posterior ethmoid region with bony destruction at the orbital apex. Examination of the nose revealed a large pale swelling far back in the middle meatus from which large quantities of mucus were aspirated by needle. The man's signs were entirely due to a large mucocoele of the posterior ethmoid region which was cured by an intranasal ethmoidectomy and a drainage operation.

This patient illustrates the need for local examination before any expensive and potentially dangerous investigations are embarked upon.

Intranasal signs are not always found in this disease but when present consist of enlarged ethmoid cells visible in the middle meatus, which may be pressed tightly against the middle turbinate. Occasionally the only abnormality in the nose is a deviated nasal septum to the side of the lesion, together with a narrow airway and a middle turbinate pressed closely to the lateral nasal wall. Nasal polyposis was present in two patients and may have been a contributory factor in obstructing the sinuses.

The presence and direction of the proptosis may be of considerable help in localizing a lesion. A mass at the orbital apex tends to produce a directly forwards proptosis, whilst lesions further forward in the fronto-ethmoidal complex produce a lateral, downwards and forwards proptosis, similar to that caused by lesions invading the orbit from a large frontal sinus. Ethmoidal lesions in infants produce a proptosis which is characteristically lateral, forwards and upwards. All this helps to distinguish fronto-ethmoidal mucocoeles from the lesions within the antrum or lacrimal sac with which they are frequently confused. The lacrimal sac lies so far forwards in the orbit that swellings within it rarely produce displacement of the globe; but if such displacement occurs the globe is moved upwards, laterally, and if anything backwards, whilst antral tumours displace the globe upwards.

\section{Radiology}

Radiology is an extremely useful aid to diagnosis in sinus mucocoeles as long as views of the paranasal sinuses are taken. In nine of our ten patients, radiological examination revealed disease of the sinuses and on six occasions the radiologist was able to make a correct diagnosis of mucocoele. However, it should be emphasized that, unless views of 
the sinuses are asked for, radiology is of little help. Two patients in our series had carotid angiography performed before radiographs were taken of the paranasal sinuses and others had specialized views of the orbit and standard antero-posterior and lateral views of the skull, none of which was of any help, weeks or months before sinus views were asked for. They should be a standard feature of the examination of any patient presenting with unilateral proptosis.

\section{Conclusions}

Mucocoeles of the frontal and ethmoidal sinuses are an uncommon cause of unilateral proptosis, but they have characteristic features which enable a diagnosis to be established without undue difficulty.

(1) The length of history before presentation helps to distinguish the lesion from Graves's disease, for in the latter eye signs become bilateral within 6 months even if the initial lesion is confined to one side.

(2) The direction of the proptosis downwards, forwards, and laterally helps to distinguish the lesion from disease of the lacrimal sac in which the displacement is in an upwards and backwards direction. It also helps to distinguish it from Graves's disease in which proptosis is purely forwards and is frequently associated with motor problems of the upper eyelid. This also helps to distinguish it from disease of the lacrimal gland, an incorrect diagnosis which is made surprisingly often.

(3) A palpable cystic swelling is frequently present in the upper and medial margin of the orbit, although confusion may arise with the fast-growing malignancy in the same area, which often has a similar soft and cystic feeling.

(4) Lack of an acute inflammatory swelling helps to distinguish a mucocoele from a ruptured sinus or acute pyocoele of the sinuses.

(5) Mucocoeles are the only cause of a long-standing proptosis which fluctuates in size, becoming more marked with the common cold.

(6) The lack of orbital motor palsies is characteristic of this disease and helps to distinguish it from malignant disease invading the orbit and particularly at the apex.

(7) A previous history of sinusitis is frequently given and is of diagnostic help.

(8) The examination of the nose often reveals swollen ethmoidal cells or obstruction to the affected lateral wall of the nose by a deviated nasal septum or a large middle turbinate.

(9) The characteristic radiological features of a mucocoele are of considerable value in establishing a diagnosis, as long as views of the paranasal sinuses are taken. Standard views of the skull and orbit are of no value.

In all our patients, diagnosis would have been established earlier if, at the time of initial presentation, local examination of the nose, palpation of the orbit, and radiological views of the paranasal sinuses had been undertaken. It is our view that a patient with a unilateral proptosis should not be submitted to expensive and often unpleasant investigations until such a local examination has been made. The most common cause of unilateral proptosis is disease of the paranasal sinuses. We feel that all patients with this presenting symptom should be referred to a rhinologist for nasal examination. Mucocoele of the sinuses is a benign readily treatable lesion and, as such, should always be thought of in this group of patients. 


\section{Summary}

The findings in ten patients with fronto-ethmoidal mucocoeles presenting with unilateral proptosis are discussed.

The length of history, direction of the proptosis, and presence of a palpable orbital swelling are considered to be valuable clues to diagnosis.

The importance of intranasal examination and sinus radiology as preliminary investigations is emphasized.

\section{REFERENCES}

Gerber (1909). Cited by Howarth (1924).

HoWARTH, W. G. (1921). Lancet, 2, 744. (1924). J. Laryng., 39, 265.

Lambert, V. F. (1955). Proc. roy. Soc. Med., 48, 79.

LANGENBECK (1820). Cited by Vail (1931).

Rollet (1909). In "Encylopédie française d'ophtalmologie", ed. F. Lagrange and E. Valude, vol. 8, p. 588. Doin, Paris.

TURNER, A. LOGAN (1907). Edinb. med. J., n.s. 22, 396, 481.

VAIL, D. T. (1931). Amer. J. Ophthal., 14, 202. 